\title{
Blood Flow after Endovascular Repair in the Aortic Arch: A Computational Analysis
}

\author{
Theodorus M. van Bakel, MD ${ }^{1,2}$ Rodrigo M. Romarowski, PhD ${ }^{1,3}$ Simone Morganti, $\mathrm{PhD}^{3}$ \\ Joost A. van Herwaarden, MD, PhD ${ }^{2}$ Frans L. Moll, MD, PhD ${ }^{2}$ Hector W. de Beaufort, MD $^{1,2}$ \\ Massimiliano M. Marrocco-Trischitta, MD, $\mathrm{PhD}^{1}$ Francesco Secchi, MD, PhD ${ }^{4}$ Michele Conti, $\mathrm{PhD}^{3}$ \\ Ferdinando Auricchio, $\mathrm{PhD}^{3}$ Santi Trimarchi, MD, $\mathrm{PhD}^{1,5}$
}

\footnotetext{
1 Thoracic Aortic Research Center, IRCCS-Policlinico San Donato, University of Milan, Milan, Italy

${ }^{2}$ Department of Vascular Surgery, University Medical Center Utrecht, Utrecht, The Netherlands

${ }^{3}$ Department of Civil Engineering and Architecture, University of Pavia, Pavia, Italy

${ }^{4}$ Department of Radiology, IRCCS-Policlinico San Donato,

San Donato Milanese, Italy

${ }^{5}$ Department of Biomedical Sciences for Health, University of Milan, Milan, Italy
}

\begin{abstract}
Address for correspondence Santi Trimarchi, MD, PhD, Thoracic Aortic Research Center, IRCCS-Policlinico San Donato, Via Morandi 30, 20097 San Donato Milanese (MI), San Donato Milanese, Italy (e-mail: Santi.Trimarchi@grupposandonato.it).
\end{abstract}

AORTA 2018;6:81-87.

\begin{abstract}
Keywords

- thoracic endovascular aortic repair

- blood flow

- displacement force

Background The benefits of thoracic endovascular aortic repair (TEVAR) have encouraged stent graft deployment more proximally in the aortic arch. This study quantifies the hemodynamic impact of TEVAR in proximal landing zone 2 on the thoracic aorta and the proximal supra-aortic branches.

Methods Patients treated with TEVAR in proximal landing zone 2 having available preoperative and 30-day postoperative computer tomography angiography and phasecontrast magnetic resonance imaging data were retrospectively selected. Blood flow was studied using patient-specific computational fluid dynamics simulations.

Results Four patients were included. Following TEVAR in proximal landing zone 2, the mean flow in the left common carotid artery (LCCA) increased almost threefold, from $0.21(0.12-0.41) \mathrm{L} / \mathrm{min}$ to $0.61(0.24-1.08) \mathrm{L} / \mathrm{min}(+294 \%)$. The surface area of the LCCA had not yet increased commensurately and therefore maximum flow velocity in the LCCA increased from $44.9(27.0-89.3) \mathrm{cm} / \mathrm{s}$ to $72.6(40.8-135.0) \mathrm{cm} / \mathrm{s}(+62 \%)$. One of the patients presented with Type lb endoleak at 1-year follow-up. The displacement force in this patient measured $32.1 \mathrm{~N}$ and was directed dorsocranial, perpendicular to the distal sealing zone. There was a linear correlation between the surface area of the stent graft and the resulting displacement force $(p=0.04)$.

Conclusion TEVAR in proximal landing zone 2 alters blood flow in the supra-aortic branches, resulting in increased flow with high flow velocities in the LCCA. High displacement forces were calculated and related to stent graft migration and Type I endoleak during 1-year follow-up.
\end{abstract}

received

May 24, 2017

accepted after revision

November 5, 2018
DOI https://doi.org/

10.1055/s-0039-1683771.

ISSN 2325-4637.
Copyright $\odot 2018$ by Thieme Medical Publishers, Inc., 333 Seventh Avenue, New York, NY 10001, USA.

Tel: +1(212) 584-4662.

\section{License terms}

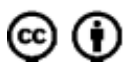




\section{Introduction}

Thoracic endovascular aortic repair (TEVAR) has decreased perioperative mortality and morbidity in treatment of diseases of the descending thoracic aorta. ${ }^{1,2}$ The benefits of endovascular treatment have encouraged most vascular surgeons to deploy stent grafts also more proximally into the aortic arch. Despite the less invasive character of TEVAR in the aortic arch, stroke remains an important complication associated with significant morbidity and mortality. ${ }^{3}$

Early on in the adoption of TEVAR as first-line treatment, a classification for preoperative planning of TEVAR in the aortic arch was proposed, identifying four proximal landing zones in the ascending aorta and aortic arch: zone 0 to $3 .{ }^{4}$ Stent graft deployment in zones 0,1 , and 2 determines the coverage of one or more proximal supra-aortic branches, which is generally managed by creating an extra-anatomical bypass, redirecting blood flow.

TEVAR in zone 2 is frequently accompanied by a left carotidto-subclavian bypass and is performed in one-third or more of patients undergoing TEVAR. ${ }^{5}$ The benefits of embolization or revascularization of the left subclavian artery (LSA) are still subject of debate.,7

The hemodynamic effects of TEVAR in zone 2 are not well understood. Zone 2 deployment is generally considered safe. ${ }^{7}$ However, there is an increased risk of stroke ${ }^{8}$ and Type I endoleak ${ }^{9}$ compared with deployment in the descending thoracic aorta, distally to the origin of the LSA. Calculation of the hemodynamic effects of stent graft deployment in zone 2 may provide additional information to help understand the pathophysiology of these complications. In this setting, computational fluid dynamics (CFD) simulations can be performed to calculate the blood flow in patient-specific models of the aorta. The aim of this study was to use this powerful tool to quantify the impact of TEVAR in zone 2 on blood flow in the aorta and supra-aortic branches.

\section{Methods}

\section{Patient Selection}

The database of IRCCS Policlinico San Donato was retrospectively quarried for patients who underwent zone 2 TEVAR for thoracic aortic diseases between 2013 and 2016. Patients who had available preoperative and 30-day postoperative computer tomography angiography (CTA) and phase-contrast magnetic resonance imaging (PC-MRI) data were included. Clinical data at 30-day and 1-year follow-up were noted. This study was approved by the local ethics committee, which waived the need for patients' informed consent due to the retrospective nature of the analysis and the use of anonymized data.

\section{Medical Imaging}

The standard CTA protocol for aortic imaging at our institution includes a 64-multislice computed tomography (CT) scan (SOMATOM Definition AS, Siemens, Germany) after administration of a contrast agent (Iomeron 370, Bracco, Milan, Italy). Image reconstructions were done with $1 \mathrm{~mm}$ slice thickness. CTA imaging data were used for measurement of the surface areas of the proximal supra-aortic branches and constructing the models for CFD simulations.

The protocol for PC-MRI in patients with aortic pathologies at our institution uses a 1.5 Tesla MRI scanner (MAGNETOM Aera, Siemens, Erlangen, Germany). Flow measurements were taken at the level of the ascending aorta, brachiocephalic trunk (BCT), left common carotid artery (LCCA), LSA, and descending aorta, and were used to set the inflow and outflow boundary conditions for the CFD simulations.

\section{Computational Fluid Dynamics Simulations}

Three-dimensional models of the thoracic aorta were constructed from CTA imaging data using the Vascular Modeling Toolkit level set segmentation (VMTK software suite version 1.3). The models included the ascending aorta, aortic arch, proximal part of the supra-aortic branches, and descending thoracic aorta. Once the models were segmented, a computational mesh was created to follow the fluid domain. The surface was smoothed with a Taubin algorithm to avoid shrinking.

CFD simulations were performed to retrieve blood flow velocity, pressure, and wall shear stress fields using previously described techniques. ${ }^{10}$ Boundary conditions were imposed in a patient-specific manner. In particular, flow profiles as extracted from each patient's pre- and postoperative PC-MRI were imposed at the ascending aorta. On the outflow sections, classic three-element Windkessel circuits were applied to mimic the compliance and resistance of the distal vascular bed. ${ }^{11,12}$ The Windkessel models were calibrated to match the patient-specific blood pressure, from brachial artery cuff measurement, and flow measurements from PC-MRI. Convergence of velocity and pressure data are a requirement to obtain meaningful CFD simulation results. ${ }^{12}$ Therefore, CFD simulations of six cardiac cycles were performed, and results from the last cardiac cycle were used. Post-processing was done using open source software ParaView version 4.1.0 (Kitware Inc, Los Alamos National Laboratory, NM).

\section{Outcome Measures}

CFD simulations were performed using preoperative and 30day postoperative imaging data. Mean flow was calculated in the ascending aorta, BCT, LCCA, LSA, and descending aorta. Blood flow through the ascending aorta, proximal supra-aortic branches, and descending aorta was calculated before and after intervention.

Flow velocity measurements were taken just distal to the origin of the proximal supra-aortic branches on the center lumen line.

Hemodynamic displacement forces acting on the surface of the stent graft were calculated from postoperative CFD simulation results (Eq. 1). ${ }^{13,14}$

$$
D F=\int p d A+\int \tau d A
$$

The displacement force (DF) in Newton is calculated by the sum of the pressure $(p)$ and wall shear stress $(\tau)$ on the surface area of the stent graft $(d A)$ in systolic peak. 
Table 1 Patient characteristics

\begin{tabular}{|l|l|l|l|l|}
\hline Patient & Age $(\mathrm{y})$ & Sex & Disease & Stent graft dimensions $(\mathrm{mm})$ \\
\hline 1 & 48 & $\mathrm{M}$ & TAA & $28-24-150$ \\
\hline 2 & 65 & $\mathrm{M}$ & PAU & $42-42-100$ \\
\hline 3 & 74 & $\mathrm{M}$ & TAA & Combined 44-44-200/46-46-200/46-46-150 \\
\hline 4 & 81 & $\mathrm{M}$ & TAA & Combined 42-42-200/46-46-200/46-46-150 \\
\hline
\end{tabular}

Abbreviations: M, male; PAU, penetrating aortic ulcer; TAA, thoracic aortic aneurysm.

Stent graft dimensions are presented in the following order: proximal diameter-distal diameter-length.
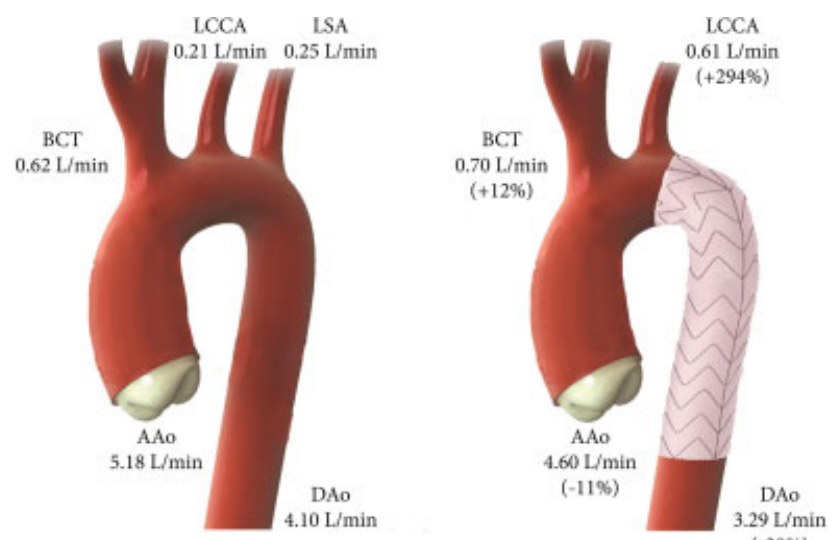

Fig. 1 Blood flow following TEVAR in zone 2. Mean flows in the ascending aorta, proximal supra-aortic branches, and descending aorta before TEVAR in zone 2 and after 30-day follow-up. The difference is given within brackets. AAo, ascending aorta; BCT, brachiocephalic trunk; DAo, descending aorta; LCCA, left common carotid artery; LSA, left subclavian artery.

\section{Statistical Analysis}

Data were analyzed using SPSS statistics version 24 (IBM, Armonk, NY). Continuous data are reported as mean values with the range given between brackets. Differences between the pre- and postoperative results are reported in percentages. Correlations were calculated using the Pearson's correlation coefficient. Statistical significance was assumed at $p<0.05$.

\section{Results}

\section{Patient Characteristics}

Four patients were selected ( - Table 1 ). All patients were treated with TEVAR in zone 2 using Medtronic Valiant stent grafts
(Medtronic Vascular, Santa Rosa, CA) and were submitted to a left carotid-to-subclavian bypass within the same procedure.

At the 30-day postoperative visit, clinical follow-up was uneventful for all patients. At 1-year follow-up, patient 4 presented with a Type Ib endoleak. Patients 1, 2, and 3 were free of complications.

\section{Mean Flows}

-Fig. 1 shows the mean flows before TEVAR and after 30day follow-up; data are given in - Table 2. Before TEVAR, the mean flow in the ascending aorta was 5.18 (3.69-6.31) $\mathrm{L} / \mathrm{min}$. Mean flow in the BCT was $0.62(0.54-0.75) \mathrm{L} / \mathrm{min}$, in the LCCA $0.21(0.12-0.41) \mathrm{L} / \mathrm{min}$, in the LSA 0.25 (0.17-0.33) $\mathrm{L} / \mathrm{min}$, and in the descending aorta 4.10 (2.25-5.26) L/min.

Following TEVAR, the mean flow in the LCCA increased almost threefold $(+294 \%)$, whereas blood flow in the ascending aorta and descending aorta decreased $(-11 \%$ and $-20 \%$, respectively).

\section{Surface Areas of Proximal Supra-aortic Branches}

The surface areas of the proximal supra-aortic branches were measured on preoperative and 30-days postoperative CT images. Data are given in - Table 3. Before TEVAR, the surface area of the BCT was $3.16(0.95-6.09) \mathrm{cm}^{2}$, the surface area of the LCCA was $0.94(0.39-1.44) \mathrm{cm}^{2}$, and the surface area of the LSA was $1.61(0.75-2.9) \mathrm{cm}^{2}$. At 30-day follow-up, the surface area of the BCT did not change $(+1 \%)$. The surface area of the LCCA increased by $9 \%$.

\section{Flow Velocities}

Data of maximum flow velocity in the proximal supra-aortic branches are given in - Table 4. Before TEVAR, maximum flow

Table 2 Blood flow before and after TEVAR in zone 2

\begin{tabular}{|c|c|c|c|c|c|c|c|c|c|}
\hline \multirow[t]{2}{*}{ Patient } & \multicolumn{3}{|c|}{ Before TEVAR } & \multirow[t]{2}{*}{ LSA } & \multirow[t]{2}{*}{ DAo } & \multicolumn{4}{|c|}{ After TEVAR } \\
\hline & AAo & BCT & LCCA & & & AAo & $\mathrm{BCT}$ & LCCA & DAo \\
\hline 1 & 6.31 & 0.54 & 0.19 & 0.33 & 5.26 & 6.64 & 1.21 & 1.08 & 4.35 \\
\hline 2 & 5.98 & 0.67 & 0.12 & 0.17 & 5.01 & 4.61 & 0.57 & 0.24 & 3.81 \\
\hline 3 & 4.74 & 0.54 & 0.12 & 0.24 & 3.85 & 4.53 & 0.72 & 0.76 & 3.05 \\
\hline 4 & 3.69 & 0.75 & 0.41 & 0.28 & 2.26 & 2.62 & 0.30 & 0.37 & 1.95 \\
\hline Mean & 5.18 & 0.62 & 0.21 & 0.25 & 4.10 & 4.60 & 0.70 & 0.61 & 3.29 \\
\hline
\end{tabular}

Abbreviations: AAo, ascending aorta; BCT, brachiocephalic trunk; DAo, descending aorta; LCCA, left common carotid artery; LSA, left subclavian artery; TEVAR, thoracic endovascular aortic repair.

Blood flow measurements are presented in $\mathrm{L} / \mathrm{min}$. 
Table 3 Surface area of the proximal supra-aortic branches before and after TEVAR in zone 2

\begin{tabular}{|l|l|l|l|l|l|}
\hline \multirow{2}{*}{ Patient } & \multicolumn{2}{|l|}{ Before TEVAR } & \multirow{2}{*}{ LSA } & \multicolumn{2}{l|}{ After TEVAR } \\
\cline { 2 - 3 } \cline { 6 - 6 } & BCT & LCCA & & BCT & LCCA \\
\hline 1 & 0.95 & 0.39 & 0.75 & 1.05 & 0.57 \\
\hline 2 & 2.22 & 0.67 & 0.82 & 2.10 & 0.76 \\
\hline 3 & 3.40 & 1.27 & 1.95 & 3.53 & 1.30 \\
\hline 4 & 6.09 & 1.44 & 2.90 & 6.06 & 1.46 \\
\hline Mean & 3.16 & 0.94 & 1.61 & 3.19 & 1.02 \\
\hline
\end{tabular}

Abbreviations: BCT, brachiocephalic trunk; LCCA, left common carotid artery; LSA, left subclavian artery; TEVAR, thoracic endovascular aortic repair. Surface areas were measured just distal to the origin from the aortic arch and are given in $\mathrm{cm}^{2}$.

Table 4 Maximum flow velocity in the proximal supra-aortic branches

\begin{tabular}{|l|l|l|l|l|l|}
\hline \multirow{2}{*}{ Patient } & \multicolumn{2}{|l|}{ Before TEVAR } & \multirow{2}{*}{ LSA } & \multicolumn{2}{l|}{ After TEVAR } \\
\cline { 2 - 3 } \cline { 6 - 6 } & BCT & LCCA & & BCT & LCCA \\
\hline 1 & 104.4 & 89.3 & 105.3 & 106.7 & 134.9 \\
\hline 2 & 36.6 & 27.0 & 25.8 & 33.7 & 40.8 \\
\hline 3 & 35.9 & 33.8 & 45.9 & 28.3 & 71.4 \\
\hline 4 & 14.1 & 29.3 & 13.3 & 8.9 & 43.2 \\
\hline Mean & 47.8 & 44.9 & 47.6 & 44.5 & 72.6 \\
\hline
\end{tabular}

Abbreviations: BCT, brachiocephalic trunk; LCCA, left common carotid artery; LSA, left subclavian artery; TEVAR, thoracic endovascular aortic repair. Maximum flow velocity before TEVAR in zone 2 and after 30-day follow-up was measured just distal to the origin from the aortic arch and given in $\mathrm{cm} / \mathrm{s}$.

velocity in the BCT was $47.9(14.1-104.4) \mathrm{cm} / \mathrm{s}$, in the LCCA $44.9(27.0-89.3) \mathrm{cm} / \mathrm{s}$, and in the LSA $47.6(13.3-105.3) \mathrm{cm} / \mathrm{s}$.

Following TEVAR, maximum flow velocity in the LCCA increased by $+62 \%$. - Fig. 2 presents a mapping of the peak systolic flow profiles in the proximal supra-aortic branches of patient 3 before and after TEVAR.

\section{Displacement Forces}

The magnitude of the displacement force acting on the stent grafts ranged from 12.2 to $32.1 \mathrm{~N}$. The displacement force vector was directed dorsocranial in all patients (-Fig. $\mathbf{3}$ ).

Patient 4 presented with Type Ib endoleak at 1-year follow-up. The center of the distal end of the stent graft migrated $17.1 \mathrm{~mm}$ dorsocranial in the direction of the displacement force (-Fig. 4).

The magnitude of the displacement force had a linear correlation with the surface area of the stent graft $(p=0.04),(-$ Fig. 5). Although the total surface area of the stent graft in patient 3 was not as large as in patient 4 , the magnitude of the displacement force was similar. This is caused by the higher blood pressure in patient 3 compared with patient 4 . Blood pressure measurements from CFD simulation results are reported in -Table 5 .

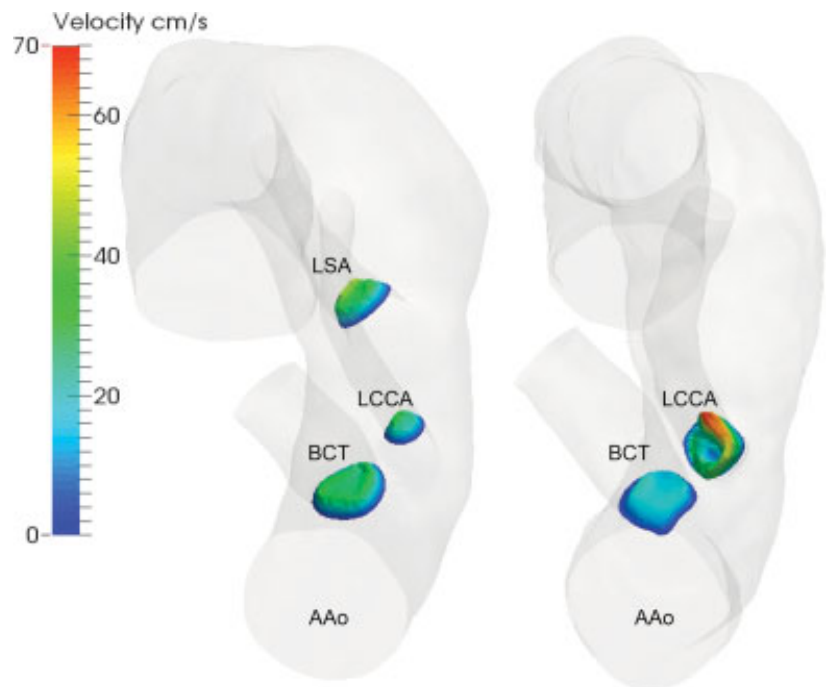

Fig. 2 Velocity contours in the proximal supra-aortic branches. Peak flow velocity is mapped just distal to the origin of the proximal supraaortic branches. AAo, ascending aorta; BCT, brachiocephalic trunk; LCCA, left common carotid artery; LSA, left subclavian artery.

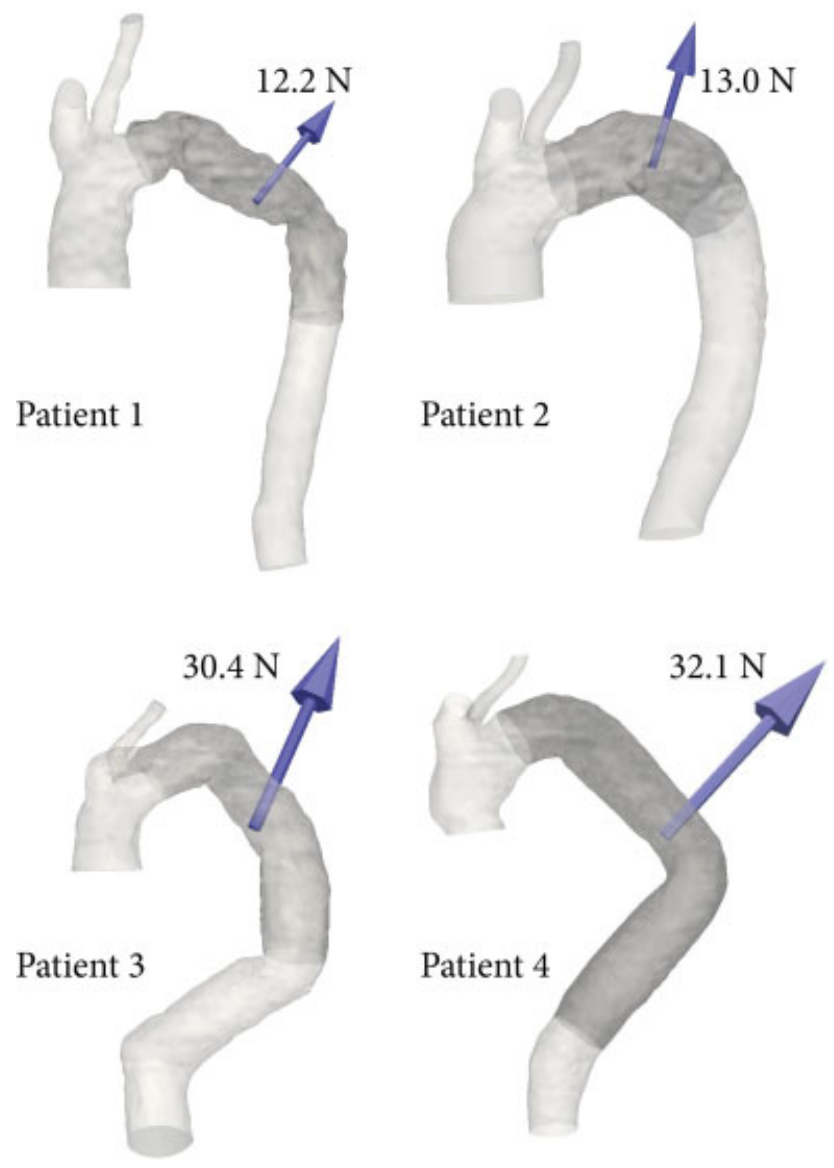

Fig. 3 Displacement forces acting on the stent grafts. The displacement force vectors are shown in all four patients. The contours of the stent grafts are outlined in dark gray. The magnitude of the displacement force is given in Newton. 

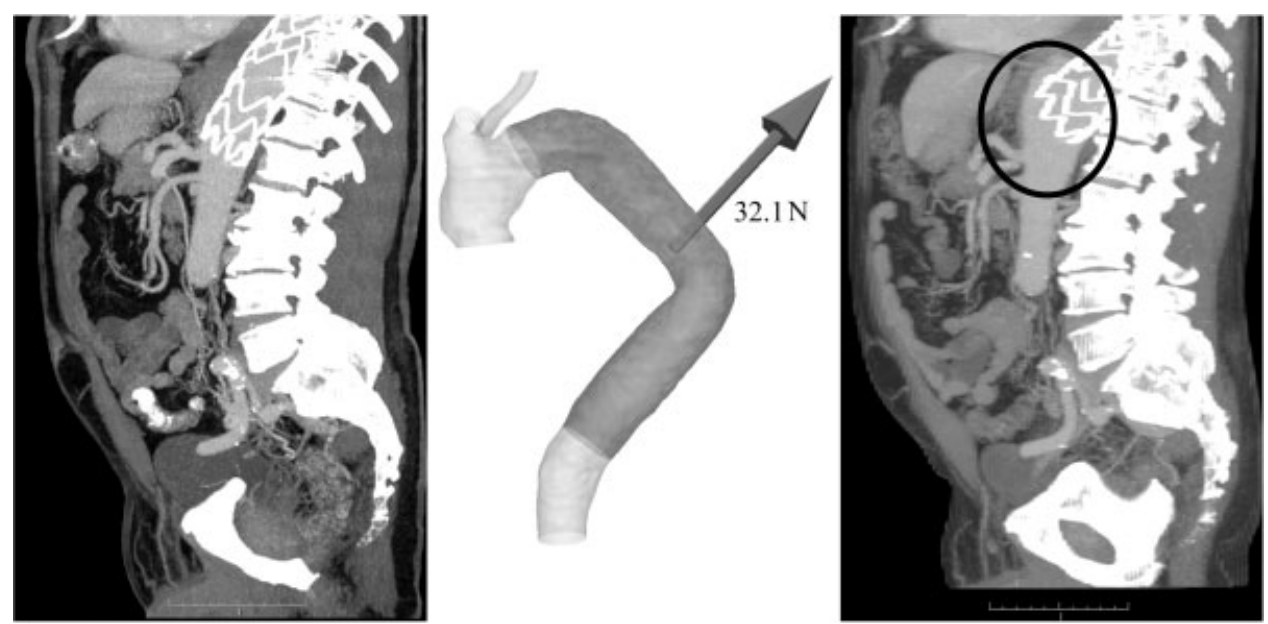

Fig. 4 Displacement force and Type Ib endoleak in patient 4. On the left, a sagittal CT image of the postoperative situation is shown. In the middle, the postoperative model with the calculated displacement force vector is presented. On the right, a sagittal CT image at 1-year follow-up is shown, where proximal migration of the stent graft and resulting Type lb endoleak was found (marked with circle).

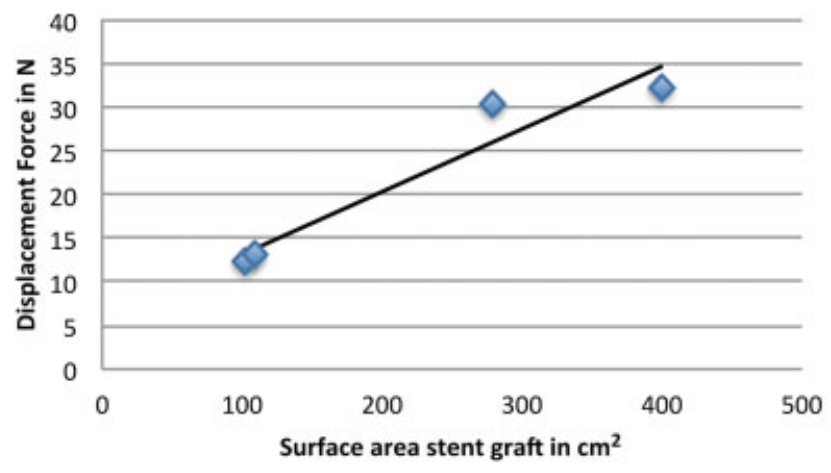

Fig. 5 Stent graft surface area and resulting displacement force. The surface areas of the stent grafts are plotted against the peak systolic displacement forces. A trend line is added visualizing the linear correlation.

\section{Discussion}

In this study, we used patient-specific CFD simulations to quantify some of the hemodynamic effects of TEVAR in zone 2 on blood flow in the aorta and the supra-aortic branches. Our results show that blood flow predominantly changed in the proximal LCCA, where flow increased almost threefold. The vessel size of the LCCA did not change commensurately at 30-day follow-up, and consequently flow velocity increased.

Little is known about the effects of TEVAR in zone 2 on blood flow in the carotid arteries. Our results show that blocking the LSA and redirecting blood flow to the left arm via the LCCA and left carotid-to-subclavian bypass increases flow velocities in the proximal LCCA. This is likely to induce arterial remodeling in the proximal LCCA during follow-up. ${ }^{15}$ The correlation between flow alterations and intimal thickening and stenosis has been investigated extensively. ${ }^{16}$ Moreover, increased flow velocities induce elevated endothelial shear stresses, which are not only a triggering factor for arterial remodeling but also atherosclerotic plaque formation and inflammatory processes. ${ }^{17,18}$ Besides inducing arterial remodeling, the outflow tract of the left carotid-to-subclavian bypass can be a relatively low-resistance path compared with the distal LCCA, especially if a stenosis is present distal to the bypass, resulting in subclavian steal from the LCCA. Further research is warranted to study these scenarios and the impact of different treatment options, such as the use of a branched stent graft versus left carotid-to-subclavian bypass, on blood flow in the carotid and vertebral arteries following zone 2 TEVAR.

Previous studies showed the relationship between displacement forces and stent graft migration in the abdominal aorta. ${ }^{19}$

Table 5 Blood pressure measurements from CFD simulation results

\begin{tabular}{|c|c|c|c|c|c|c|}
\hline \multirow[t]{2}{*}{ Patient } & \multicolumn{2}{|c|}{ Before TEVAR } & \multirow[t]{2}{*}{ Mean } & \multicolumn{2}{|c|}{ After TEVAR } & \multirow[t]{2}{*}{ Mean } \\
\hline & Systolic & Diastolic & & Systolic & Diastolic & \\
\hline 1 & 131 & 58 & 82 & 127 & 66 & 87 \\
\hline 2 & 110 & 49 & 80 & 108 & 56 & 80 \\
\hline 3 & 117 & 61 & 89 & 114 & 65 & 88 \\
\hline 4 & 94 & 62 & 77 & 106 & 55 & 77 \\
\hline Mean & 113 & 58 & 82 & 114 & 61 & 83 \\
\hline
\end{tabular}

Abbreviations: CFD, computational fluid dynamics; TEVAR, thoracic endovascular aortic repair.

Blood pressures measurements were taken from CFD simulation results at the ascending aorta and are given in $\mathrm{mm} \mathrm{Hg}$. 
Compared with the abdominal aorta, the displacement force is significantly larger and directed cranial in the thoracic aorta. ${ }^{14,20}$ Stent graft size is a key determinant of the magnitude of the displacement force. ${ }^{21}$ Our results confirm these findings. The patient in whom we had calculated the highest displacement force, measuring $31.1 \mathrm{~N}$, presented with Type Ib endoleak after 1-year follow-up. The displacement force calculated in this patient lies within the range of pullout forces described by Rahmani et al. ${ }^{22}$ Interestingly, we calculated a similar displacement force in patient 3, measuring $30.4 \mathrm{~N}$; however, no migration or endoleak was found in this case. We hypothesize that the direction of the displacement force being perpendicular to the distal sealing zone might have attributed to the stent graft being pulled out of its original position.

Further investigation of the hemodynamic displacement force and the complex of compensatory radial and frictional forces, securing stability of the stent graft in the sealing zones, is required. In the future, these mechanisms could potentially be taken into account in preoperative planning for TEVAR, thus reducing the risk of Type I endoleak.

\section{Limitations}

A limitation of the CFD simulations performed for this study is the rigid wall assumption. More accurate simulations would include fluid structure interaction, for which computational costs are significantly higher. ${ }^{23}$

We simulated only flow through arteries for which we had patient-specific flow measurements; therefore, we did not include the coronary and distal carotid arteries in our models.

Our CFD simulation results showed increased flow velocities in the LCCA. Future prospective studies that focus on the impact of TEVAR in zone 2 on cerebral blood flow should also include flow measurements more distal in the internal and external carotid arteries and in the vertebral arteries.

The small number of patients included in this study limits the impact of the results. Although clear trends were found, studies consisting of a larger study population are required to confirm our findings.

\section{Conclusion}

TEVAR in proximal landing zone 2 alters blood flow in the supra-aortic branches, resulting in increased flow with high flow velocities in the LCCA. Our results warrant further investigation of cerebral blood flow following TEVAR in the aortic arch. The use of large stent grafts in the thoracic aorta results in high displacement forces, increasing the risk of stent graft migration and Type I endoleak during follow-up.

\section{Funding \\ None.}

\section{Conflict of Interest}

The authors declare no conflict of interest related to the article.

\section{Acknowledgments}

None.

\section{References}

1 Goodney PP, Travis L, Lucas FL, et al. Survival after open versus endovascular thoracic aortic aneurysm repair in an observational study of the Medicare population. Circulation 2011;124(24): 2661-2669

2 Gopaldas RR, Huh J, Dao TK, et al. Superior nationwide outcomes of endovascular versus open repair for isolated descending thoracic aortic aneurysm in 11,669 patients. J Thorac Cardiovasc Surg 2010;140(05):1001-1010

3 Gutsche JT, Cheung AT, McGarvey ML, et al. Risk factors for perioperative stroke after thoracic endovascular aortic repair. Ann Thorac Surg 2007;84(04):1195-1200, discussion 1200

4 Ishimaru S. Endografting of the aortic arch. J Endovasc Ther 2004; 11(Suppl 2):II62-II71

5 Patterson B, Holt P, Nienaber C, Cambria R, Fairman R, Thompson $M$. Aortic pathology determines midterm outcome after endovascular repair of the thoracic aorta: report from the Medtronic Thoracic Endovascular Registry (MOTHER) database. Circulation 2013;127(01):24-32

6 Sobocinski J, Patterson BO, Karthikesalingam A, Thompson MM. The effect of left subclavian artery coverage in thoracic endovascular aortic repair. Ann Thorac Surg 2016;101(02): 810-817

7 Contrella BN, Sabri SS, Tracci MC, et al. Outcomes of coverage of the left subclavian artery during endovascular repair of the thoracic aorta. J Vasc Interv Radiol 2015;26(11):1609-1614

8 Ullery BW, McGarvey M, Cheung AT, et al. Vascular distribution of stroke and its relationship to perioperative mortality and neurologic outcome after thoracic endovascular aortic repair. J Vasc Surg 2012;56(06):1510-1517

9 Kanaoka Y, Ohki T, Maeda K, Baba T. Analysis of risk factors for early type I endoleaks after thoracic endovascular aneurysm repair. J Endovasc Ther 2017;24(01):89-96

10 van Bogerijen GHW, Auricchio F, Conti M, et al. Aortic hemodynamics after thoracic endovascular aortic repair, with particular attention to the bird-beak configuration. J Endovasc Ther 2014;21 (06):791-802

11 Alimohammadi M, Agu O, Balabani S, Díaz-Zuccarini V. Development of a patient-specific simulation tool to analyse aortic dissections: assessment of mixed patient-specific flow and pressure boundary conditions. Med Eng Phys 2014;36(03): 275-284

12 Vignon-Clementel IE, Figueroa CA, Jansen KE, Taylor CA. Outflow boundary conditions for 3D simulations of non-periodic blood flow and pressure fields in deformable arteries. Comput Methods Biomech Biomed Engin 2010;13(05):625-640

13 Morris L, Delassus P, Walsh M, McGloughlin T. A mathematical model to predict the in vivo pulsatile drag forces acting on bifurcated stent grafts used in endovascular treatment of abdominal aortic aneurysms (AAA). J Biomech 2004;37(07): 1087-1095

14 Figueroa CA, Taylor CA, Chiou AJ, Yeh V, Zarins CK. Magnitude and direction of pulsatile displacement forces acting on thoracic aortic endografts. J Endovasc Ther 2009;16(03): 350-358

15 Nichols W, O'Rourke M, Vlachopoulos C. The nature of flow of a liquid. In: Nichols W, O'Rourke M, Vlachopoulos C, eds. McDonald's Blood Flow in Arteries: Theoretical, Experimental and Clinical Principles. 6th ed. New York, NY: CRC Press; 2011

16 Grant EG, Benson CB, Moneta GL, et al. Carotid artery stenosis: gray-scale and Doppler US diagnosis-Society of Radiologists in Ultrasound Consensus Conference. Radiology 2003;229(02): 340-346

17 Cecchi E, Giglioli C, Valente S, et al. Role of hemodynamic shear stress in cardiovascular disease. Atherosclerosis 2011;214(02): 249-256

18 Morbiducci U, Kok AM, Kwak BR, Stone PH, Steinman DA, Wentzel $\mathrm{JJ}$. Atherosclerosis at arterial bifurcations: evidence for the role of 
haemodynamics and geometry. Thromb Haemost 2016;115(03): 484-492

19 Figueroa CA, Yeh V, Taylor CA, Gorrepati ML, Zarins CK. In vivo displacement force (DF) is higher in patients who experience aortic endograft migration: A 3D computational analysis. J Vasc Surg 2010;51:93S

20 Krsmanovic D, Koncar I, Petrovic D, Milasinovic D, Davidovic L, Filipovic N. Computer modelling of maximal displacement forces in endoluminal thoracic aortic stent graft. Comput Methods Biomech Biomed Engin 2014;17(09):1012-1020
21 Prasad A, To LK, Gorrepati ML, Zarins CK, Figueroa CA. Computational analysis of stresses acting on intermodular junctions in thoracic aortic endografts. J Endovasc Ther 2011;18(04): 559-568

22 Rahmani S, Grewal IS, Nabovati A, Doyle MG, Roche-Nagle G, Tse LW. Increasing angulation decreases measured aortic stent graft pullout forces. J Vasc Surg 2016;63(02):493-499

23 Brown AG, Shi Y, Marzo A, et al. Accuracy vs. computational time: translating aortic simulations to the clinic. J Biomech 2012;45 (03):516-523 